\title{
ON COUPLED FIELDS IN STRATIFIED PLASMAS WITH TENSOR PRESSURE PERTURBATIONS
}

\author{
R. Burman, W. C. Anderson
}

(Czech. J. Phys. B23 (1973), 685-797)

P. $786^{17} \quad u_{0}^{2} \equiv 5 p_{0} / 3 m N_{0}$

$$
\nabla \cdot \boldsymbol{p}=\theta \nabla p+\eta \nabla \times \nabla \times \mathbf{v}
$$

$$
-\nabla \times \nabla \times \boldsymbol{H}+\frac{\nabla \varepsilon}{\varepsilon} \times(\nabla \times \boldsymbol{H})+k_{0}^{2} \varepsilon \boldsymbol{H}=\frac{e}{\mathrm{i} \omega m U}\left[\nabla \times(\nabla \cdot \boldsymbol{p})-\frac{\nabla \varepsilon}{\varepsilon} \times(\nabla \cdot \boldsymbol{p})\right]
$$

$$
\nabla \cdot(\nabla \cdot \mathbf{p})-\frac{\nabla X}{X} \cdot(\nabla \cdot \mathbf{p})+\frac{\omega^{2} U}{u_{0}^{2}} p=e N_{0} \nabla . \mathbf{E} .
$$

$$
\nabla \cdot(\nabla \cdot \boldsymbol{p})-\frac{\nabla(X \varepsilon)}{X \varepsilon} \cdot(\nabla \cdot \boldsymbol{p})+\frac{\omega^{2}}{u_{0}^{2}} U \varepsilon p=\frac{i \omega m}{e} X \frac{\nabla \varepsilon}{\varepsilon} \cdot(\nabla \times \boldsymbol{H}) .
$$

in eqs $(15),(16) \frac{(U \varepsilon)^{\prime}}{U \varepsilon}$ should read $\frac{\varepsilon^{\prime}}{\varepsilon}$

(18)

$$
\text { the first line should read } \begin{array}{lllll}
0 & 0 & 0 & -1
\end{array}
$$

$$
\alpha \text { should read } \propto
$$

$$
2 q_{1,3}^{2}=1+\frac{U X}{\alpha} \pm \delta^{1 / 2}
$$

the last term should read $\ln \left(X \delta^{1 / 2} \phi\right)$ 